\title{
An Update on XCOV25: GD358
}

\author{
J. L. Provencal ${ }^{1}$, H. L. Shipman ${ }^{1}$, and The WET TEAM ${ }^{2}$ \\ ${ }^{1}$ Mt. Cuba Observatory and the University of Delaware \\ Dept. of Physics and Astronomy, Newark, DE 19716 \\ ${ }^{2}$ www.physics.udel.edu/darc/wet/
}

\begin{abstract}
We present a preliminary report on $436.1 \mathrm{hrs}$ of nearly continuous high-speed photometry on the pulsating DB white dwarf GD358 acquired with the Whole Earth Telescope, in concert with the Delaware Asteroseismic Research Center (DARC) during May 12 to June 16, 2006.
\end{abstract}

\section{Introduction}

Asteroseismology of stellar remnants is traditionally thought of as the study of interior structure of pulsating white dwarfs and subdwarfs as revealed by global stellar oscillations. The light from stellar sources, be it detected using a $0.6 \mathrm{~m}$ or a $10 \mathrm{~m}$ telescope, originates from their surfaces. Stellar oscillations contain information about the interior of the star through which they have traveled, allowing a view beneath the photosphere. In the case of white dwarfs, the individual pulsations are temperature variations arising from nonradial g-modes. Asteroseismology allows us to retrieve information about basic physical parameters, including mass, rotation rate, and internal composition. This information (see for example: Winget et al. 1991, Winget et al. 1994, Kepler et al. 2003, Kanaan et al. 2005) provides important insights into a wide range of fields, from stellar formation and evolution, the chemical evolution in our galaxy, the age of the galactic disk, and the physics of Type la supernovae.

Asteroseismology is now expanding its focus to investigate problems that at first consideration may not be best suited for these techniques. Convection remains one of the largest sources of theoretical uncertainty in our understanding of stars. Our lack of understanding leads to considerable systematic theoretical 
uncertainties in such important quantities as the ages of massive stars (DiMauro et al. 2003) and the temperatures and cooling ages of white dwarfs (Wood 1992). Montgomery (2005) shows how precise observations of the light curves of variable stars can be used to characterize the convection zone in a particular star. Montgomery bases his approach on important analytical and numerical precursor calculations (Brickhill 1992, Goldreich \& Wu 1999, Wu 2001). The method is based on three assumptions: 1) the flux perturbations are sinusoidal below the convection zone, 2) the pulsations can be treated to first order as if they were radial, and 3) the convective turnover time is short compared with the pulsations so the convection zone can be assumed to respond instantaneously. This approach can observationally determine the convective time scale $\tau_{0}$, a temperature dependence parameter $\mathrm{N}$, and, together with an independent $\mathrm{T}_{\text {eff }}$ determination, the classical convective efficiency parameter (the mixing length ratio) $\alpha$.

In concert with the Delaware Asteroseismic Research Center (Provencal et al. 2005), we organized a WET run in May of 2006 with GD358 as the prime target (XCOV25). Our purpose was twofold: 1) obtain at least 5 hours of high signal to noise photometry from a large telescope and 2) accurately identify the frequencies, amplitudes and phases present in GD358's current pulsation spectrum. We fulfilled both of our goals. In the following, we will provide a preliminary overview of the data set and reduction procedures and present a preliminary list of identified modes, combination frequencies, and multiplet structure.

\section{The Observations}

XCOV 25 spans May 12 to June 14. Nineteen observatories participated in the run, contributing a total of 88 runs (a complete list of participants and observing runs can be found at www.physics.udel.edu/darc/wet/XCov25/xcov25.html). We obtained $436.1 \mathrm{hrs}$ of observations, achieving $73 \%$ coverage during the main portion of the run.

Recent WET runs (examples include Kanaan et al. 2005) comprise a mixture of CCD and PMT observations, and XCOV25 is no exception. CCDs were employed at sixteen observatories, and 3-channel PMT photometers at the remaining sites. We attempted to minimize bandpass issues by using CCDs with similar chips and equipping each CCD with a BG40 or S8612 filter to normalize wavelength response and reduce extinction effects. The bi-alkali photomultiplier tubes are blue sensitive, so no filters were required. We also made every attempt to observe the same comparison star at each site.

Standard procedure for a WET run is for observers to transfer observations to headquarters for analysis at the end of each night. In the past, CCD 
observers completed initial reductions (bias, flat, and dark removal) at their individual sites, performed preliminary photometry, and transferred the result to WET headquarters. For XCOV25, the majority of participants using CCD photometers transferred their raw images, enabling headquarters to funnel data through a uniform reduction pipeline. The few sites unable to transfer images nightly performed preliminary reductions on site using the same procedures as those at headquarters, and sent their images at a later date.

The PMT data were reduced using the WET standard prescription developed by Nather et al. (1990). CCD data reduction followed the pipeline described by Kanaan et al. (2002). Figure 1 presents the lightcurve from the central 2 weeks of XCOV25 observations.

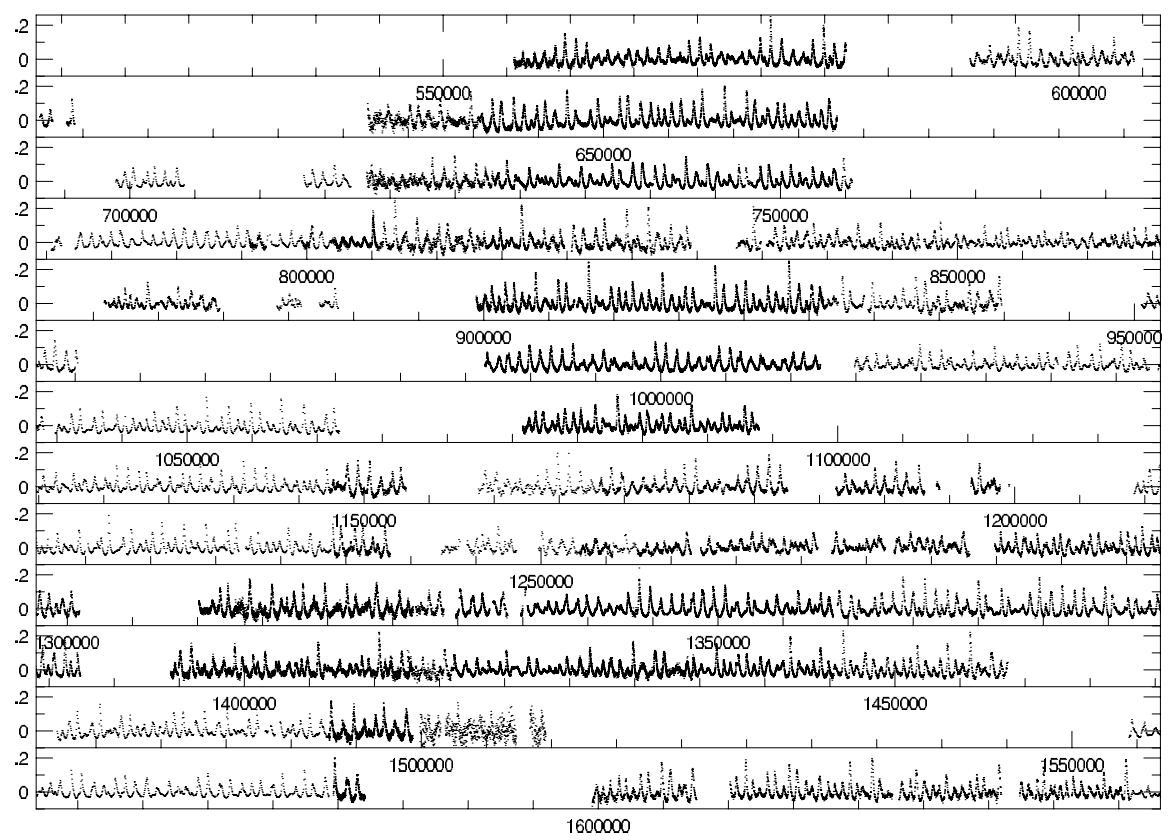

Figure 1: Central 2 weeks of XCOV25 light curve. Each panel is 1 day of observations.

Figure 2 presents the Fourier Transform (FT) of the entire data set. We carried out multi-frequency analysis using the Period04 software package (Lentz 2004). The basic method involves identifying the largest amplitude peak in the FT, subtracting that sinusoid from the original lightcurve, recomputing the FT, examining the residuals, and repeating the process. This technique is fraught with peril, as it is possible for overlapping spectral windows to conspire to produce alias amplitudes larger than any real signal. Our final identifications result 
from a simultaneous nonlinear least squares fit of 130 frequencies, amplitudes, and phases, some of which are labelled in Figure 2. We employed this procedure to identify 130 frequencies satisfying our criteria of amplitudes $4 \sigma$ above the average noise.

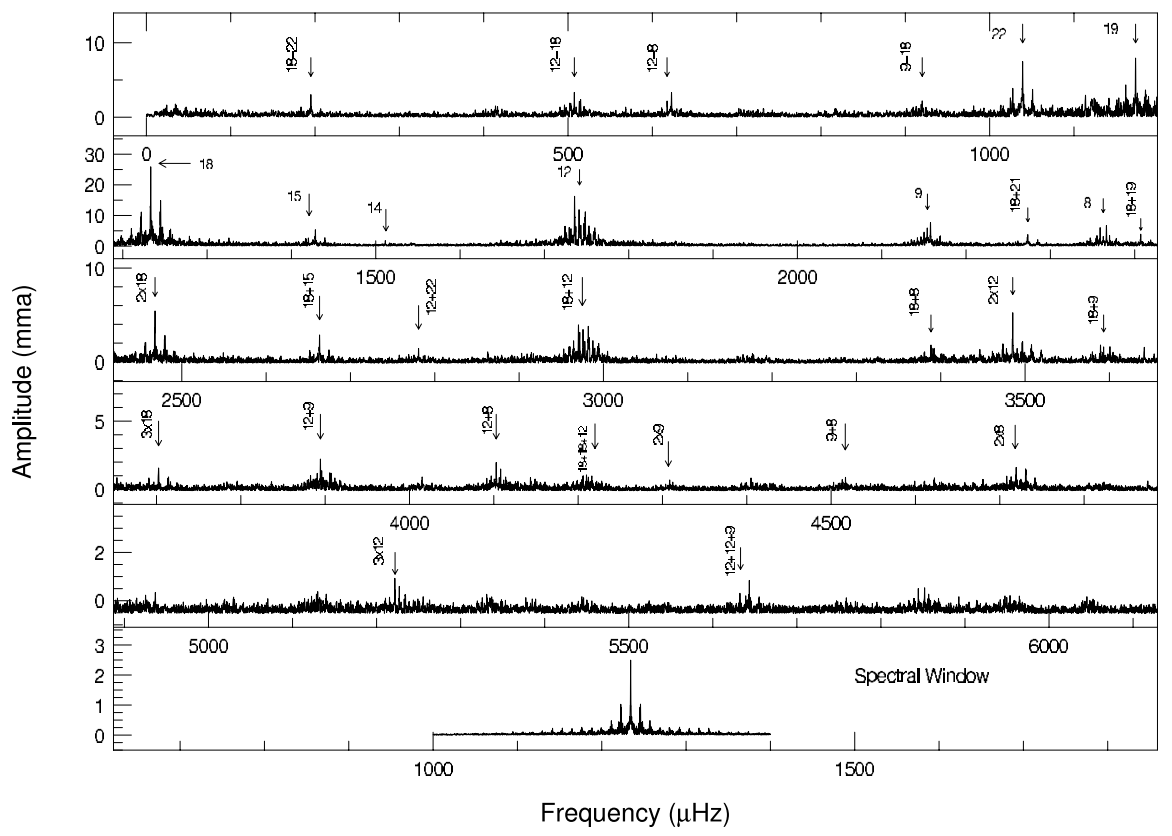

Figure 2: Fourier Transform of GD358 (XCOV25)

The data set contains a significant fraction of overlapping data. We experimented with the effects of overlapping data on the FT by computing FTs with 1) all data included, 2) no overlapping data, where we kept those data with higher signal to noise ratio, and 3) weighting the overlapping lightcurves by telescope aperture size. Figure 3 presents a comparison between the spectral window of the entire run and the central 14 days shown in Figure 1. We found no significant differences between the spectral window or FTs of overlapping versus nonoverlapping versus weighted data.

\section{The Fourier Transform}

Table 1 lists a sample of preliminary frequency identifications, due to space considerations. The complete list can be found in Provencal et al. (2008). We adopt the identifications of Winget et al. (1994), but must emphasize that 


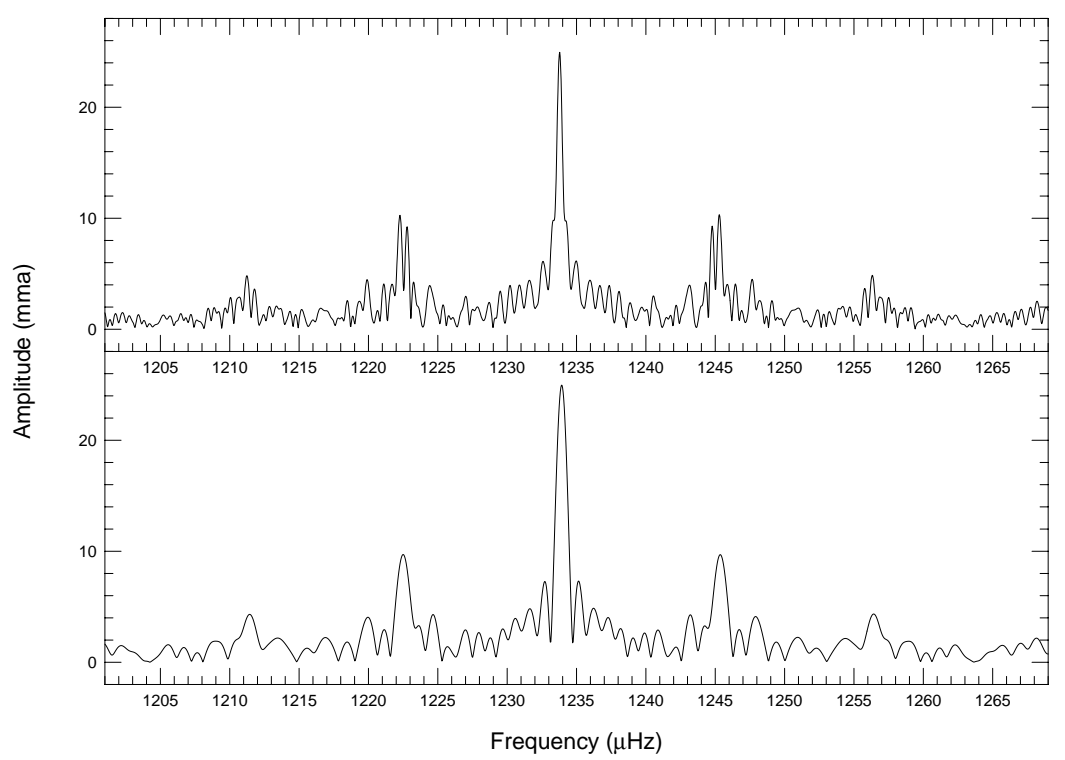

Figure 3: Comparison of the spectral window of the entire WET run with that from the main portion of the run given in Figure 1.

while we are confident from previous work that the dominant modes are $l=1$, the actual $k$ identification cannot be observationally determined and may not correspond precisely to the values given here.

The dominant mode in 2006 is $k=18(1234.124 \mu \mathrm{Hz}, 810.291 \mathrm{~s})$ with an average amplitude of $24.04 \mathrm{mma}$. Mode 18 is detected in previous observations (see Kepler et al. 2003) but not as the dominant frequency. The previously dominant $k=15$ and 17 modes have greatly diminished amplitudes, and we do not detect $k=16$ or 13 . Perhaps the greatest surprise is the appearance of prominent power near the predicted value for $k=12$, a region of the FT previously devoid of significant peaks. This mode was detected in 1990, 1994, 1996, and 2000 but never at an amplitude about $1 \mathrm{mma}$. We do not detect the suspected $l=2$ mode at $1255.4 \mu \mathrm{Hz}$ noted in Kepler et al. (2003). We also do not find $k=7$ at $2675.5 \mu \mathrm{Hz}$. (Kepler et al. 2003) suggest that this mode may have been excited to visibility via resonant coupling with $k=17$ and 16 . Since 
$k=17$ and 16 do not have significant amplitude in 2006 , it follows that $k=7$ would not be detected.

GD358's FT contains a rich distribution of combination frequencies, from simple harmonics to fourth order combinations, some of which are given in Table 1. Most of these combinations are exact to within statistical uncertainties. Combination peaks, whose frequencies are linear combinations (both sums and differences) of 2 or more mode components, are typically observed in large amplitude pulsators (Dolez et al. 2006, Thompson et al. 2003). The general consensus on the origin of these peaks (Brassard et al. 1995, Yeates et al. 2005) argues that they are indicative of nonlinear distortions induced by the propagating medium, in this case the convection zone. The convection zone acts as a nonlinear filter, varying its depth in response to the pulsations, and distorting the original sinusoidal variations. We expect the amplitudes of the observed combinations, and the number associated with a given parent mode, to be a function of the flux intensity of the mode(s) involved.

\section{Comparison with Other Observing Seasons}

Figure 4 presents a sampling of GD358's FT for seasons spanning 1990 to 2006. We pause here to reflect on our previous mention of spectral windows and alias patterns. The 1990, 1991, 1994, 2000, and 2006 FTs are from WET runs, and the other seasons are single site, obtained from McDonald Observatory. Comparison of the FTs and corresponding spectral windows dramatically illustrates the power of WET to reduce alias artifacts.

A simple visual comparison of the FTs in Figure 4 illustrates the simultaneous simplicity and complexity of GD358. While large amplitude peaks are always confined between $1000-1800 \mu \mathrm{Hz}$, and individual modes appear in the same general location over the years, the distribution and amplitude of excited modes varies. Figure 5 shows the frequency of the largest amplitude peak in each FT, from 1982 to 2007. 
Table 1: A Sample of Identified Frequencies Frequency $\mu \mathrm{Hz}$ Amplitude (mma) Not $\pm 0.001 \mu \mathrm{Hz}$ $\pm 0.07 \mathrm{mma}$

$\begin{array}{ccc}195.685 & 2.70 & 18-21 \\ 617.431 & 2.03 & 18 / 2 \\ 1039.076 & 7.94 & \mathrm{k}=21 \\ 1173.015 & 7.24 & \mathrm{k}=19 \\ 1222.946 & 4.30 & \mathrm{k}=18 \\ 1228.792 & 5.06 & \mathrm{k}=18 \\ 1234.124 & 24.03 & \mathrm{k}=18 \\ 1239.511 & 4.93 & \mathrm{k}=18 \\ 1245.220 & 4.90 & \mathrm{k}=18 \\ 1429.210 & 5.63 & \mathrm{k}=15 \\ 1512.141 & 1.80 & \mathrm{k}=14 \\ 1736.311 & 16.35 & \mathrm{k}=12 \\ 1737.962 & 5.60 & \mathrm{k}=12 \\ 1741.666 & 11.01 & \mathrm{k}=12 \\ 1743.738 & 5.60 & \mathrm{k}=12 \\ 1746.672 & 1.81 & \mathrm{k}=12 \\ 1749.083 & 10.92 & \mathrm{k}=12 \\ 1856.845 & 1.41 & \mathrm{k}=11 \\ 2150.393 & 4.10 & \mathrm{k}=9 \\ 2154.224 & 5.51 & \mathrm{k}=9 \\ 2158.074 & 7.18 & \mathrm{k}=9 \\ 2273.691 & 4.23 & 18+21 \\ 2359.053 & 5.95 & \mathrm{k}=8 \\ 2363.058 & 1.64 & \mathrm{k}=8 \\ 2366.524 & 6.60 & \mathrm{k}=8 \\ 2407.205 & 3.80 & 18+19 \\ 2468.282 & 5.19 & 2 \times 18 \\ 2663.368 & 2.95 & 18+15 \\ 2909.416 & 1.00 & 18+12 \\ 2964.917 & 1.10 & 18+12 \\ 2970.400 & 3.01 & 18+12 \\ 2972.085 & 2.82 & 18+12 \\ 2975.814 & 3.47 & 18+12 \\ 2977.885 & 1.71 & 18+12 \\ 2981.032 & 1.31 & 18+12 \\ 2981.947 & 3.53 & 18+12 \\ 2983.266 & 3.12 & 18+12 \\ 2988.643 & 1.10 & 18+12 \\ & & \\ & & \end{array}$




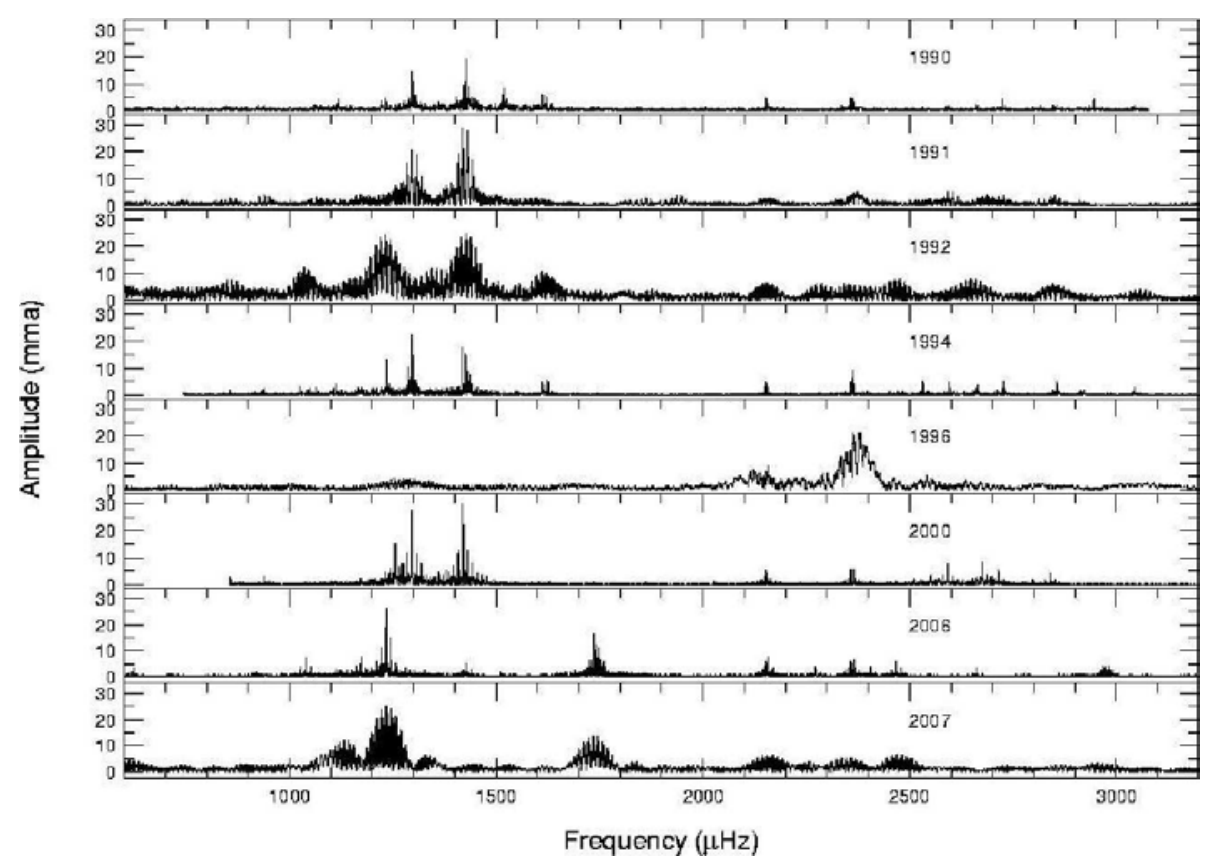

Figure 4: Comparison of GD358's FT from 1990 to 2006

\section{Multiplet Structure}

The analysis of fine structure multiplets in pulsating white dwarfs is based on the assumption that the multiplets are produced by lifting of the degeneracy of the azimuthal quantum number $m$ by rotation and/or magnetic fields. In the limit of slow rotation, we expect the observed fine structure to reflect the star's rotation rate and the spherical harmonic degree $l$ of the pulsation involved, with possible perturbations introduced by any surface magnetic field. We also expect the fine structure to remain stable over long time periods. A classic example is the prototype DO pulsator PG1159-035, which exhibits beautiful triplets, corresponding to $l=1$, and quintuplets, corresponding to $l=2$ (see Figures 5 and 6 in Winget et al. 1991). All of the multiplets of a given $l$ have the same frequency splitting, with ratio of different $l$ values very close to the expected theoretical prediction.

Figure 6 presents a "snapshot" of multiplet structure in the XCOV25 FT. Winget et al. (1994) (hereafter W94) identified triplet structure for most modes, with frequency splittings that varied with both $k$ and $m$. In 2006, the only modes exhibiting clear triplet structure with splittings in agreement 


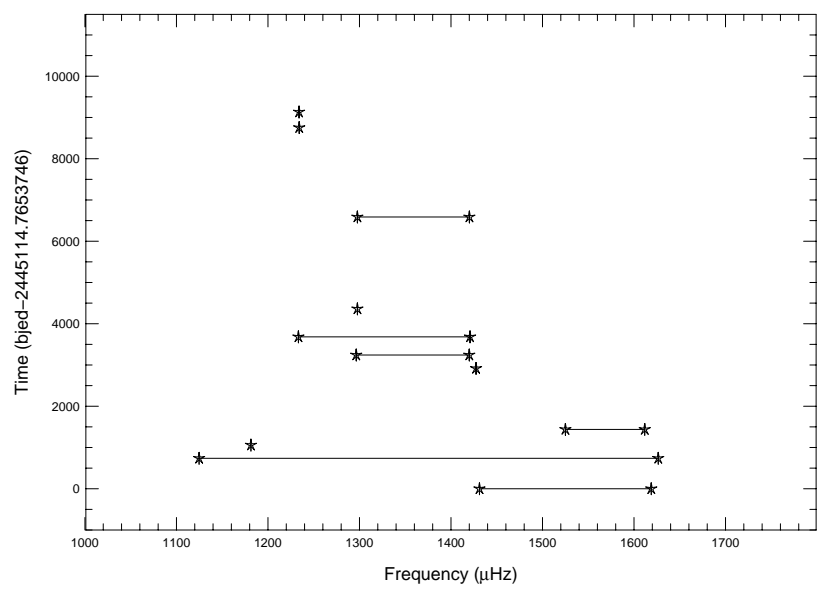

Figure 5: Frequency of the largest amplitude peak in GD358 from 1982-2007. The y axis shows time, and the $x$-axis displays frequency. The time is given in baryocentric julian ephemeris date. Tzero is 1982 . In several seasons, there are two peaks that have amplitudes within $2 \mathrm{mma}$. We have included both frequencies and joined them with a line for clarity.

with previous observations are $k=9$ and 8 . We find average multiplet splittings of 3.83 and $3.75 \mu \mathrm{Hz}$, respectively. The only other mode we detect in common with both Winget et al. (1994) and Kepler et al. (2003) that has sufficient amplitude to investigate fine structure is $k=15$, but the multiplet structure is quite different from previous reports. In 1990 (Figure 7), multiplet 15 had a reported average multiplet splitting of $6.4 \mu \mathrm{Hz}$. In 1994 , the value was $\approx 6.7 \mu \mathrm{Hz}$, and in $2000, \approx 6 \mu \mathrm{Hz}$. In $2006, k=15$ contains multiple components with a dominant splitting of $\approx 5.4 \mu \mathrm{Hz}$. The $5.4 \mu \mathrm{Hz}$ splitting also appears in $k=18$ (a quintuplet in 2006) and $k=12$. We point out that the other high $k$ modes (17, 16,14 , and 13) reported by $W 94$ to have frequency splittings of $\approx 6 \mu \mathrm{Hz}$ are not detected here.

\section{Conclusions and Speculations}

The analysis of this data set is still underway, but has already provided new insight into GD358. As is true with any new observations, the data set has 


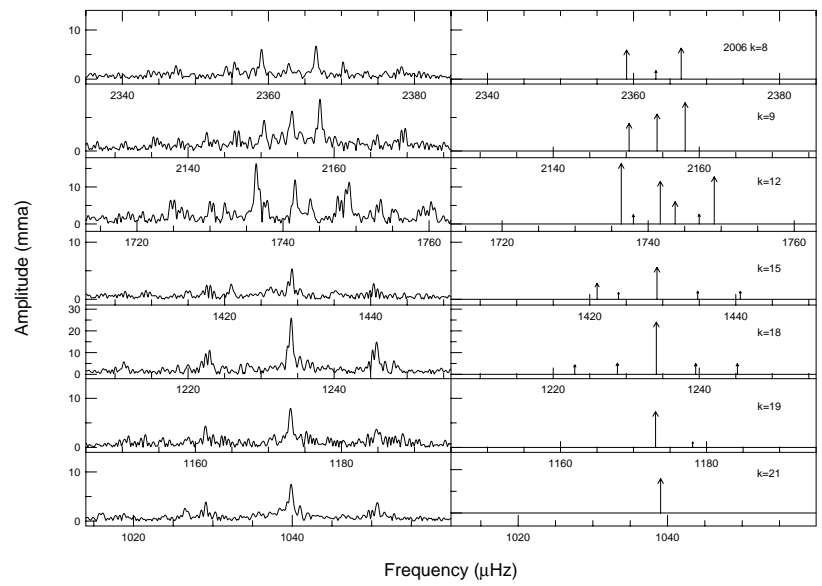

Figure 6: A "snapshot" of modes and their multiplet structure for 2006. The left panels give the actual FTs, and the right panels give the prewhitening results. Each panel spans $50 \mu \mathrm{Hz}$. Note the change in y-scale.

also raised many new questions. The role of the convection zone in nonlinear pulsators is becoming clearer. For example, convection does not play a role in the DOV pulsators, and the prototype PG1159-035's FT displays expected triplets and quintuplets corresponding to $l=1$ and 2 pulsations, and does not contain combination frequencies. Convection does play a role in GD358, and we find no evidence for stable multiplet structure here, and we do find a myriad of combination frequencies. Reviving memories of basic physics demonstrations, water in a tank will reflect off the tank walls. In a star, the bottom of the convection zone plays the role of the wall. Yet, because the star is pulsating, the convection zone is constantly changing. For $a n \mathrm{~m}=0$ mode, the poles appear to recede, but the equator does not. In other words, the convection zone does not always form a perfectly spherical reflective surface. Could this explain the difference in behavior of the various modes in GD358? Can this explain the apparent changes in mode amplitudes we observe?

We are also investigating the possible role of magnetic fields. As a simple model, one could imagine switching on a global magnetic field on a rotating, pulsating white dwarf. Our imaginary magnetic field is confined to the nondegenerate atmosphere of the white dwarf. Its influence would be strongest at 


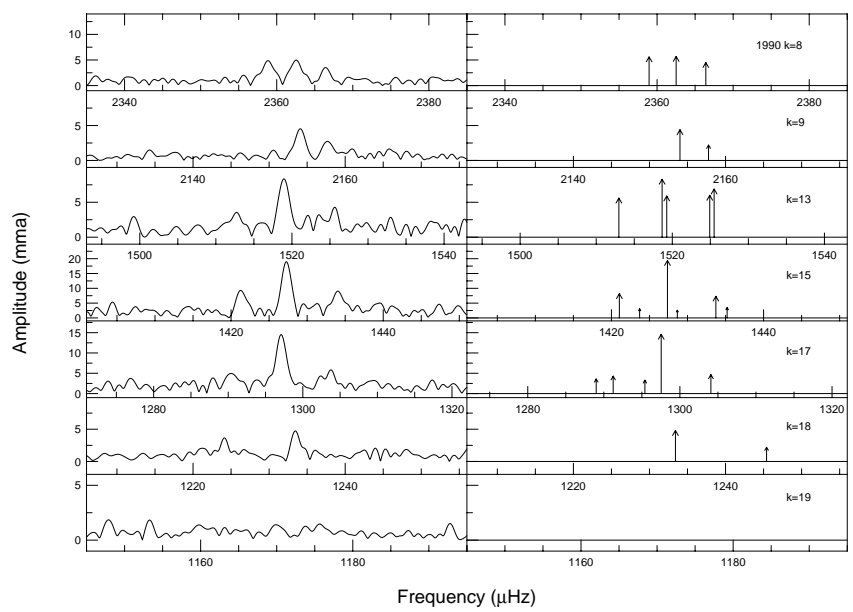

Figure 7: A "snapshot" of modes and their multiplet structure for 1990. The left panels give the actual FTs, and the right panels given the prewhitening results. Each panel spans $50 \mu \mathrm{Hz}$. Note any changes in y-scale.

the surface where the gas pressure is decreasing, but not strong enough to affect rotation or convection at the surface. If the field is a dipole, each pulsation mode could be split into $(2 l+1)^{2}$ components. For $l=1$, each mode could contain up to 9 components. Since the high $k$ modes preferentially sample the surface, could the presence of a non-aligned, variable magnetic field explain the dramatic changes in multiplet structure we observe, while the low $k$ modes, which are not influenced as strongly by the magnetic field and/or the convection zone, are left relatively unaffected?

Our continuing investigation into these questions includes a detailed analysis of multiplet structure over time, a closer look at the combination frequencies, an effort to detect a possible magnetic cycle period, and continued monitoring of GD358 and other high amplitude pulsators.

Acknowledgments. DARC acknowledges the support of the Crystal Trust Foundation and Mt. Cuba Observatory. We would also like to thank everyone involved in the network for their time and support in obtaining these observations. 


\section{References}

Brassard, P., Fontaine, G., \& Wesemael, F. 1995, ApJS, 96, 545

Brickhill, A. J. 1992, MNRAS, 259, 519

Goldreich, P., \& Wu, Y. 1999, ApJ, 511, 904

Kanaan, A., Kepler, S. O., \& Winget, D. E. 2002, A\&A, 389, 896

Kanaan, A., Nitta, A., Winget, D. E., et al. 2005, A\&A, 432, 219

Kepler, S. O., Nather, R. E., Winget, D. E., et al. 2003, A\&A, 401, 639

Lenz, P., \& Breger, M. 2004, IAU Symposium, 224, 786

Nather, R. E., Winget, D. E., Clemens, J. C., et al. 1990, ApJ, 361, 309

Montgomery, M. 2005, ApJ, 633, 1142

Provencal, J. L., Shipman, H. L., \& the Wet Team 2005, BAAS, 37, 1157

Provencal, J. L., et al. 2008, in preparation

Thompson, S. E., Clemens, J. C., van Kerkwijk, M. H., et al. 2003, ApJ, 589, 921

Winget, D. E., Nather, R. E., Clemens, J. C., et al. 1991, ApJ, 378, 326

Winget, D. E., Nather, R. E., Clemens, J. C., et al. 1994, ApJ, 430, 839

Wu, Y. 2001, MNRAS, 323, 248

Yeates, C. M., Clemens, J. C., Thompson, S. E., \& Mullally, F. 2005, ApJ, 635, 1239 


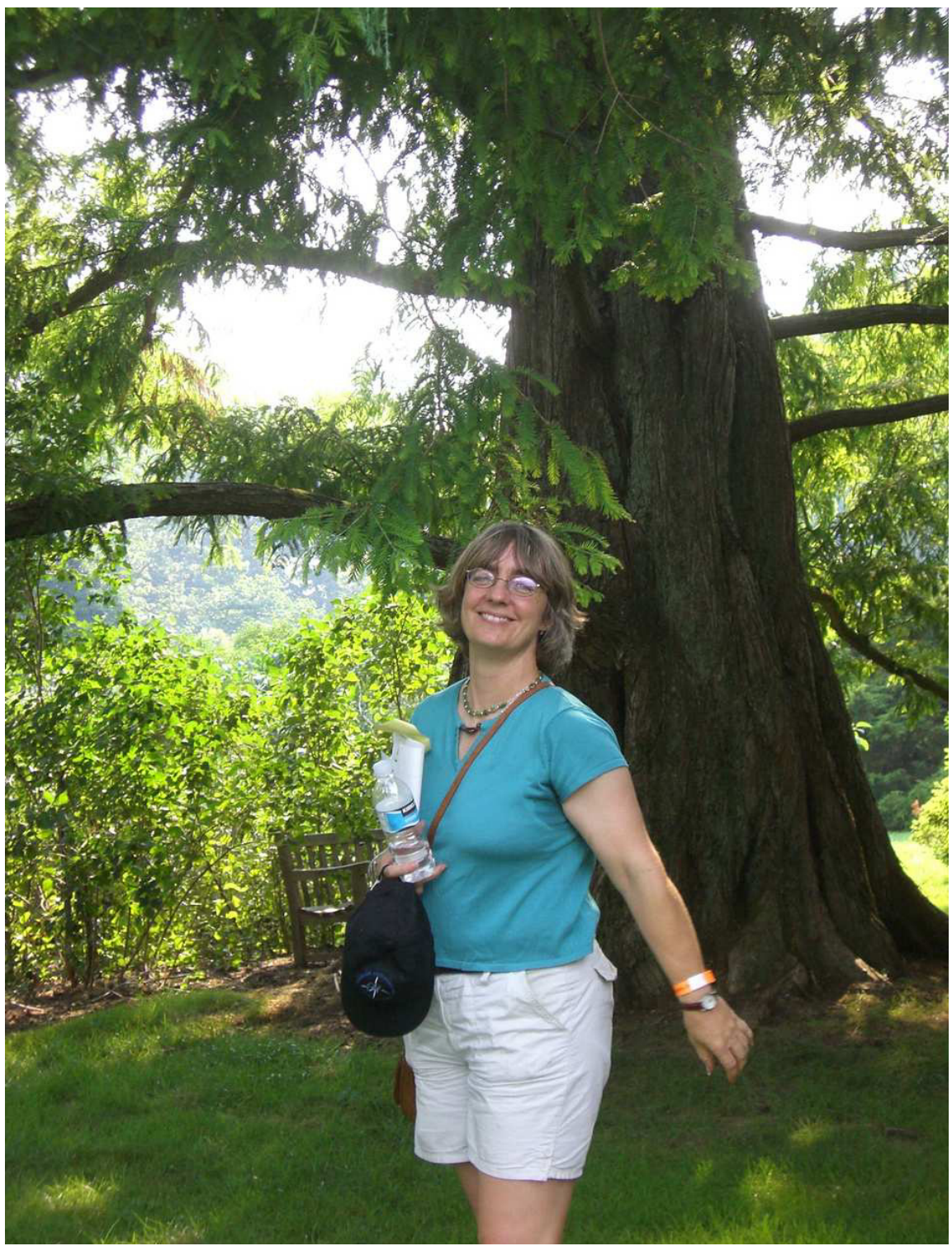

J. Provencal, the WET's director, enjoying herself at Winterthur. 